\title{
Vibrational Spectroscopy of GaN:Mg Under Pressure
}

\author{
M.D. McCluskey, K.K. Zhuravlev, M. Kneissl, ${ }^{1}$ W. Wong, ${ }^{1}$ D. Treat, ${ }^{1}$ S. Limpijumnong, ${ }^{1}$ \\ C.G. Van de Walle, ${ }^{1}$ N.M. Johnson ${ }^{1}$ \\ Department of Physics and Institute for Shock Physics, Washington State University, \\ Pullman, WA 99164-2814, U.S.A. \\ ${ }^{1}$ Xerox PARC, 3333 Coyote Hill Rd., \\ Palo Alto, CA 94304, U.S.A.
}

\begin{abstract}
The microscopic structure of $\mathrm{Mg}-\mathrm{H}$ complexes in $\mathrm{GaN}$ has been a subject of intense theoretical and experimental investigation. In order to probe the $\mathrm{Mg}-\mathrm{H}$ structure, we have studied the effect of hydrostatic pressure on the local vibrational mode (LVM) frequency. At ambient pressure, the LVM frequency is $3125 \mathrm{~cm}^{-1}$, which corresponds to a N-H stretching mode. In this study, Fourier-transform spectroscopy was performed on free-standing GaN:Mg, $\mathrm{H}$ samples in a diamond-anvil cell, with nitrogen as a pressure-transmitting fluid. The samples had been removed from their sapphire substrate by the laser-liftoff technique. The LVM frequency was measured, at liquid helium temperatures, for pressures ranging from 0 to $5 \mathrm{GPa}$. The pressure dependence of the frequency is nonlinear: first it decreases with pressure, then it increases. Comparison with first-principles calculations allows us to derive information about the microscopic structure of the Mg-H complex. The calculated stable configuration indeed gives rise to a frequency shift consistent with experiment. Based on the comparison between theory and experiment, we can exclude the bond-center configuration, which would result in a much larger pressure derivative than experimentally observed.
\end{abstract}

\section{INTRODUCTION}

$\mathrm{GaN}$ is a preferred material for optoelectronic devices such as blue-violet and UV lightemitting diodes (LEDs) and lasers [1,2]. Effective $p$-type doping of $\mathrm{GaN}$ has been a significant challenge for the fabrication of efficient devices with long operating lifetime. Metalorganic chemical vapor deposition (MOCVD) is the dominant growth technique for III-V nitride devices, with $\mathrm{Mg}$ the most common $p$-type dopant. As a result of hydrogen passivation during growth, as-grown $\mathrm{GaN}: \mathrm{Mg}$ is semi-insulating. It was shown empirically that low energy electron beam irradiation [3] or thermal annealing at temperatures above $600^{\circ} \mathrm{C}$ in an $\mathrm{N}_{2}$ ambient [4] were required to activate the $\mathrm{Mg}$ acceptors. Infrared (IR) spectroscopy was used to positively identify the $\mathrm{Mg}-\mathrm{H}$ complexes [5].

The stretch-mode frequency of hydrogen in the $\mathrm{Mg}-\mathrm{H}$ complex was calculated to be approximately $3360 \mathrm{~cm}^{-1}[6,7]$, consistent with a N-H stretching mode. The LVM was observed at $3125 \mathrm{~cm}^{-1}$ in $4 \mu \mathrm{m}$ thick epilayers of MOCVD-grown GaN:Mg [5]. Upon annealing, the peak at $3125 \mathrm{~cm}^{-1}$ decreased by a factor of two and was correlated with an increase in the conductivity. Annealed samples that are exposed to a remote deuterium plasma show a deuterium stretch mode peak at $2321 \mathrm{~cm}^{-1}$. The isotopic frequency ratio is $r=v_{\mathrm{H}} / v_{\mathrm{D}}=1.346$, which is very similar to that of $\mathrm{NH}_{3}(r=1.342)$, lending further support to the $\mathrm{N}-\mathrm{H}$ model. The details of the microscopic structure, however, have not been resolved conclusively [8]. 


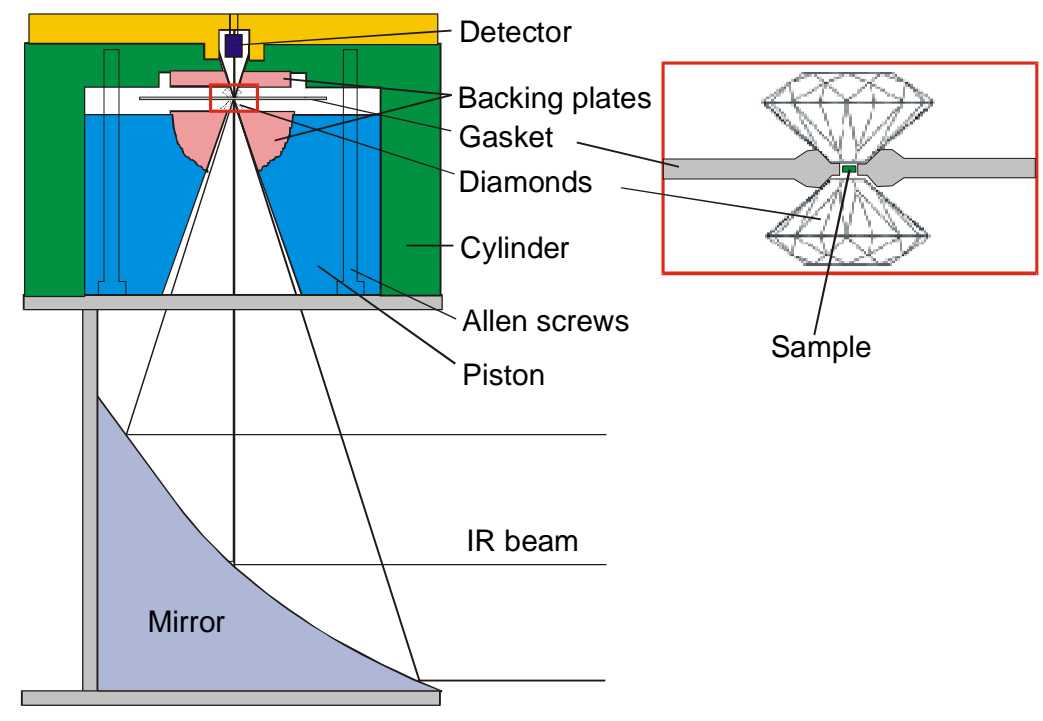

Figure 1. Cross section of DAC assembly designed for IR spectroscopy. Diamond anvils, gasket, and sample are shown in the inset.

Until recently, experimental and theoretical studies have focused on the antibonding $(\mathrm{AB})$ and bond-centered (BC) configurations. Polarized IR absorption experiments by Clerjaud et al. [9] have indicated that the $\mathrm{N}-\mathrm{H}$ bond lies at a $130^{\circ}$ angle to the $c$-axis. This angle is incompatible with the $\mathrm{AB}$ configuration, in which the $\mathrm{N}-\mathrm{H}$ bond occurs at $\sim 110^{\circ}$ to the $c$-axis. Recently, firstprinciples calculations $[7,10]$ have shown that a novel "OA" (off-axis) configuration exhibits a $134^{\circ}$ angle, in good agreement with Ref. [9]. Although the OA configuration is higher in energy than $\mathrm{AB}$ at $T=0$, it is stabilized at high temperatures due to an increase in the entropy [10].

\section{EXPERIMENTAL DETAILS}

In order to probe the $\mathrm{Mg}-\mathrm{H}$ structure, we studied the effect of hydrostatic pressure on the LVM frequency. To measure the IR spectra of semiconductors under pressure, a custom diamond anvil cell was used (Fig. 1). In this cell, force is applied by six Allen screws, which squeeze together a piston and cylinder [11]. The diamonds are supported by flat and hemispherical backing plates made from tungsten carbide. An off-axis parabolic mirror efficiently focuses the collimated IR beam onto the sample. A photoconducting Ge:Cu detector [12] is placed in close proximity to the sample, so that a large fraction of the transmitted IR light is collected. The entire assembly is placed in a Janis STVP-100 liquid-helium cryostat and kept at a temperature of 6-12 K. The low temperatures are advantageous for two reasons. First, the LVM lines sharpen with decreasing temperature. Second, the sensitive, low-noise Ge:Cu detector operates at liquid-helium temperatures.

Diamonds are transparent over a wide spectral range, although type I diamonds contain nitrogen impurities that strongly absorb from 1000 to $1500 \mathrm{~cm}^{-1}$. Type II-A diamonds, used in this study, do not contain this absorption band. However, all diamonds contain a two-photon absorption band around $2100 \mathrm{~cm}^{-1} . \mathrm{N}_{2}$, Ar, or He are typically used as the ambient, since, unlike alcohol mixtures, they are transparent to IR light. In this work, $\mathrm{N}_{2}$ was used. In $\mathrm{N}_{2}$, a vibrational mode of $\mathrm{CO}_{2}$ impurities is sensitive to pressure and was used as a precise in situ pressure 


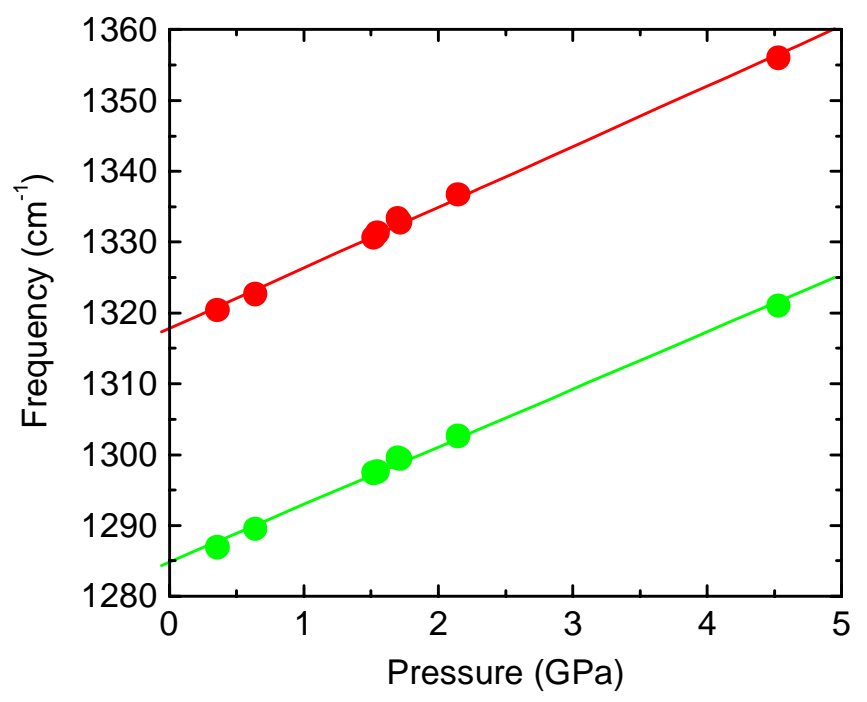

Figure 2. Pressure dependence of IR-active, two-phonon modes in GaN at a temperature of $8 \mathrm{~K}$.

calibration for these IR absorption experiments [13]. This calibration has been established up to a pressure of $7 \mathrm{GPa}$.

The sample consisted of a $1 \mu \mathrm{m}$ layer of $\mathrm{GaN}: \mathrm{Mg}, \mathrm{H}$ on a $4 \mu \mathrm{m}$ layer of nominally undoped $\mathrm{GaN}$, grown by MOCVD on a sapphire substrate. The primary difficulty in these experiments was obtaining a sufficient signal-to-noise ratio, given the very weak absorption coefficient of the $\mathrm{Mg}-\mathrm{H}$ peak. To achieve that goal, the GaN epilayer was removed from the sapphire substrate by the laser lift-off technique [14]. Several (5-10) pieces were placed in the diamond-anvil cell and subjected to pressure, using liquid nitrogen as an ambient. Given the large number or samples in the DAC, the effective sample thickness was increased, thereby increasing the absorption of the $\mathrm{Mg}-\mathrm{H}$ peak.

\section{DISCUSSION}

\section{$\underline{\text { Lattice Phonons }}$}

Strong IR absorption peaks were observed in the spectral region around $1300 \mathrm{~cm}^{-1}$. These peaks are attributed to two-phonon modes of the GaN lattice. The peak frequencies of two of these peaks are plotted as a function of pressure in Fig. 2. Linear fits to the data yield

$$
\begin{aligned}
& v_{1}=(1284.8 \pm 0.4)+(8.1 \pm 0.2) P \\
& v_{2}=(1317.8 \pm 0.4)+(8.6 \pm 0.2) P
\end{aligned}
$$

where the frequencies $v$ are in units of $\mathrm{cm}^{-1}$ and the pressure $P$ is in units of GPa. The pressure calibration was determined by $\mathrm{CO}_{2}$ molecules in the solid $\mathrm{N}_{2}$ ambient. In some experiments, however, there was not a sufficiently high concentration of $\mathrm{CO}_{2}$ to use that method. Hence, for 


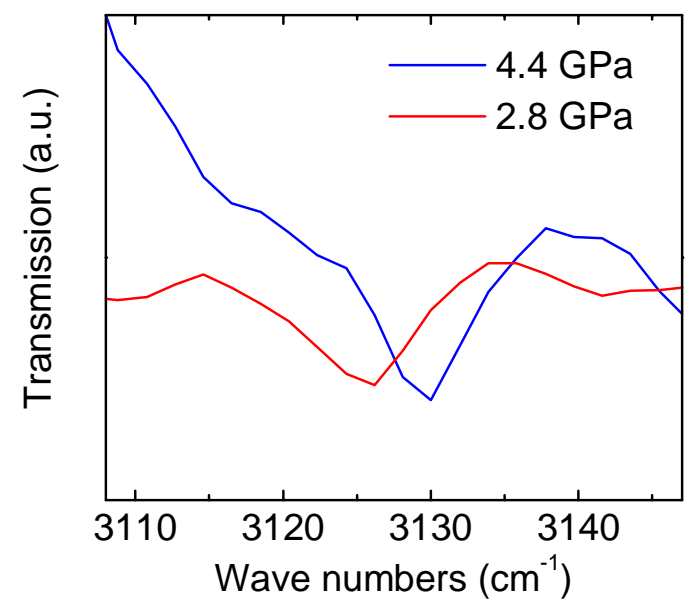

Figure 3. IR spectra of $\mathrm{GaN}: \mathrm{Mg}, \mathrm{H}$ at two different pressures, at a temperature of $8 \mathrm{~K}$.

consistency, the pressure dependence of the two-phonon peaks was used as an internal pressure calibrant, via Eqs. (1) and (2).

\section{$\underline{\text { Mg-H LVM }}$}

IR spectra of GaN:Mg,H at two different pressures are shown in Fig. 3. The LVM frequency is shown as a function of pressure in Fig. 4. The data points in Fig. 4 are a compilation of two separate experiments, in which different pieces from the same wafer were loaded into the DAC. After each spectrum was obtained, the sample was warmed to room temperature and the pressure was adjusted. The pressure was increased and decreased several times, and no hysteresis was

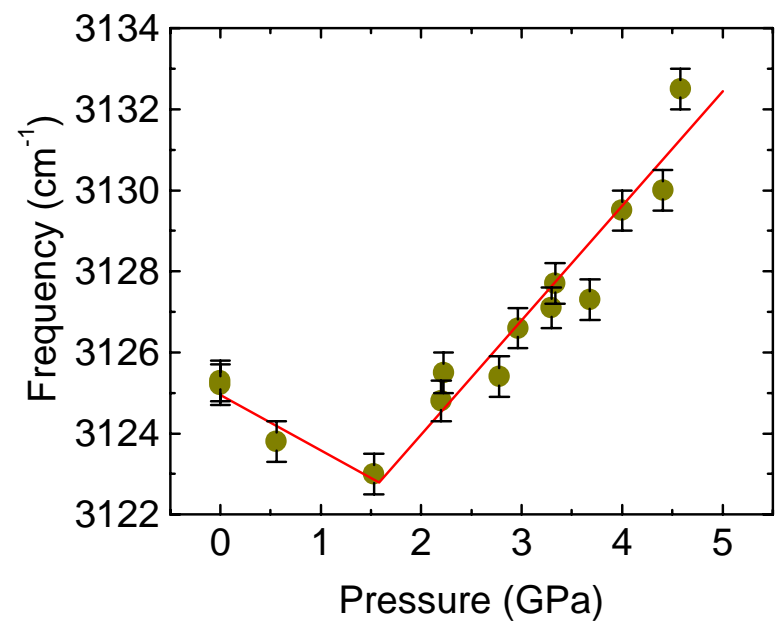

Figure 4. Pressure dependence of the Mg-H LVM frequency in $\mathrm{GaN}$ at a temperature of 8 K. 
observed. For pressures $0<P<2 \mathrm{GPa}$, the intensity of the peak was generally weaker than for $P>2 \mathrm{GPa}$. Hence, in spite of many attempts, only three reliable data points were obtained for the low-pressure range. Interestingly, the LVM frequency appears to decrease with pressure, then increase. A piecewise linear fit to the data yields:

$$
v= \begin{cases}(3125.0 \pm 0.4)-(1.4 \pm 0.4) P & P \leq 1.6 \\ (3118.3 \pm 1.1)+(2.8 \pm 0.3) P & P>1.6\end{cases}
$$

\section{Theory}

The experimental pressure shifts were compared with predictions from first-principles calculations, using density functional theory in the local density approximation and normconserving potentials with a plane-wave basis set [10]. To simulate hydrostatic pressure, the $a$ and $c$ lattice constants were each reduced by $1 \%$. Assuming a bulk modulus of $200 \mathrm{GPa}$, an isotropic strain of $1 \%$ (volume decrease of $3 \%$ ) corresponds to approximately $6 \mathrm{GPa}$. Given that value, the pressure derivatives of the LVM frequencies were calculated for hydrogen in the BC, $\mathrm{AB}$, and $\mathrm{OA}$ configurations. For the $\mathrm{BC}$ configuration (where $\mathrm{H}$ was inserted into a $\mathrm{Mg}-\mathrm{N}$ bond along the $c$-axis), the frequency shift was $15 \mathrm{~cm}^{-1} / \mathrm{GPa}$, much larger than the experimentally observed shifts in Eq. (3). Therefore, we can safely exclude BC as a candidate for the observed $\mathrm{Mg}-\mathrm{H}$ complexes. The first-principles calculations actually show that $\mathrm{BC}$ is not a stable configuration: a small displacement causes it to relax to the OA configuration. The calculated $\mathrm{AB}$ and $\mathrm{OA}$ frequency shifts were -0.3 and $1.5 \mathrm{~cm}^{-1}$, respectively. For the $\mathrm{AB}$ configuration, the $\mathrm{N}-\mathrm{H}$ bond formed an angle of $109^{\circ}$ with the $c$-axis. In the $\mathrm{AB}$ and $\mathrm{OA}$ positions, the hydrogen is not crowded by neighboring atoms, resulting in a relatively small frequency shift. The error bars for the calculated frequencies are too large to unambiguously distinguish between $\mathrm{AB}$ and OA based on the measured pressure shifts. However, we observe that the calculated result for OA agrees reasonably well with experiment for $P>1.6 \mathrm{GPa}$.

\section{CONCLUSIONS}

By measuring the pressure dependence of the GaN:Mg,H LVM and comparing with firstprinciples calculations, the $\mathrm{BC}$ configuration can be ruled out. The unresolved question is whether hydrogen is more likely to reside in the $\mathrm{AB}$ or OA configurations. The experimental pressure shift at larger pressures is more consistent with the OA configuration, but the error bars on the calculated frequencies do not allow an unambiguous identification. These uncertainties render it even more difficult to address the cause of the discontinuity in the slope of the $\mathrm{Mg}-\mathrm{H}$ LVM vs. pressure plot. A transformation between different configurations appears unlikely, since no discontinuity is observed in the frequency. Another possibility is that the nonlinear pressure dependence is due to a "buckling" of the OA hydrogen under pressure, similar to what is observed in $\mathrm{Si}: \mathrm{O}$ [15]. In future work, to test isotope effects, $\mathrm{GaN}: \mathrm{Mg}, \mathrm{D}$ samples will be investigated.

\section{ACKNOWLEDGMENTS}

This work was supported in part by NSF Grant No. DMR-9901625 and in part by AFOSR under contract \# F4920-00-C-0019. 


\section{REFERENCES}

1. S. Nakamura, Mater. Res. Soc. Symposia Proc. 482, 1145 (1998).

2. N.M. Johnson, A.V. Nurmikko, and S.P. DenBaars, Physics Today 53 (10), 31 (2000).

3. H. Amano, M. Kito, K. Hiramatsu, and I. Akasaki, Jpn. J. Appl. Phys. 28, L2112 (1989).

4. S. Nakamura, T. Mukai, M. Senoh, and N. Iwasa, Jpn. J. Appl. Phys. 31, L139 (1992).

5. W. Götz, N.M. Johnson, D.P. Bour, M.D. McCluskey, and E.E. Haller, Appl. Phys. Lett. 69, 3725 (1996).

6. J. Neugebauer and C.G. Van de Walle, Appl. Phys. Lett. 68, 1829 (1996).

7. C.J. Fall, R. Jones, P.R. Briddon, and S. Öberg, Mat.. Sci. Eng. B 82, 88 (2001).

8. W.R. Wampler, S.M. Myers, A.F. Wright, J.C. Barbour, C.H. Seager, and J. Han, J. Appl. Phys. 90, 108 (2001).

9. B. Clerjaud, D. Côte, A. Lebkiri, C. Naud, J.M. Baranowski, K. Pakula, D. Wasik, and T. Suski, Phys. Rev. B 61, 8238 (2000).

10. S. Limpijumnong, J.E. Northrup, and C.G. Van de Walle, Phys. Rev. Lett. 87, 205505 (2001). 11. G. Yu. Machavariani, M.P. Pasternak, G.R. Hearne, and G. Kh. Rozenberg, Rev. Sci. Instrum. 69, 1423 (1998).

12. Haller-Beeman Associates, Inc. (www.haller-beeman.com).

13. M.D. McCluskey, L. Hsu, L. Wang, and E.E. Haller, Phys. Rev. B 54, 8962 (1996).

14. E.A. Stach, M. Kelsch, E.C. Nelson, W.S. Wong, T. Sands, and N.W. Cheung, Appl. Phys. Lett. 77, 1819 (2000).

15. M.D. McCluskey and E.E. Haller, Phys. Rev. B 56, 9520 (1997). 